\title{
Proteolytic Lactococcus lactis and Lipolytic Enterococcus durans of Dairy Origin as Meat Functional Starter Cultures
}

\author{
Mirna Mrkonjic Fuka ${ }^{1 *}{ }_{\odot}$ \\ Ivica $\operatorname{Kos}^{2} \odot$, \\ Ana Zgomba Maksimovic ${ }^{1} \mathbb{1}$, \\ Melita Bacic ${ }^{\top} \odot$ and \\ Irina Tanuwidjaja'• \\ 'Department of Microbiology, \\ University of Zagreb, Faculty of \\ Agriculture, Svetošimunska cesta 25, \\ Zagreb, Croatia \\ 2Department of Animal Technology, \\ University of Zagreb, Faculty of \\ Agriculture, Svetošimunska cesta 25, \\ Zagreb, Croatia
}

Received: 1 July 2020 Accepted: 22 February 2021

\footnotetext{
${ }^{*}$ Corresponding author:

Phone: +38512394034

E-mail:mfuka@agr.hr
}

\section{SUMMARY}

Research background. As fermentation is an integral feature of both, dry sausage and cheese production, this has led to the evaluation of bacterial cultures Lactococcus lactis ssp. cremoris (LL8307) and Enterococcus durans (ED0207) originally isolated from artisanal Croatian hard type cheese to diversify the range of flavours of dry fermented sausages and to increase their microbiological safety. Both strains were chosen for their high or medium acidifying, proteolytic and/or lipolytic activity and bioprotective potential after step-by-step selection of wild isolates. Therefore, this study aims to evaluate the survival rate of selected starter cultures in wild boar meat sausages during the ripening period of 40 days at a local small-scale facility under artisanal conditions as well as their influence on sausage quality parameters.

Experimental approach. Safety, biotechnological and probiotic properties of twenty-three enterococcal and lactococcal isolates of dairy origin were studied. Based on the results, two best candidates were selected and added to the meat batter during the artisanal wild boar meat sausage preparation where their survival rate, effect on physicochemical, microbiological and sensorial properties and histamine content were evaluated.

Results and conclusions. As revealed by repetitive element-polymerase chain reaction (rep-PCR), native starter cultures survived up to 15 days of ripening and were either absent from (LL8307) or reduced by $80 \%$ (ED0207) in final products. The application of native starter cultures rapidly decreased $\mathrm{pH}(\mathrm{p}<0.05)$, leading to the significantly lower load of $E$. coli, coliforms and Enterobacteriaceae in ready-to-eat sausages prepared by the addition of starter cultures (3.04-3.94 log CFU/g) than in the control (3.88-5.00 log CFU/g). Analysis of hedonic test data revealed that some of the sensory traits (odour, flavour, juiciness) of treatments with starter cultures were highly liked by the higher percentage of consumers. The results suggest that these starter cultures would represent a valuable tool to improve the homogeneity of artisanal manufacture and hygienic quality of fermented sausages and can be safely used for food application.

Novelty and ccientific contribution. This is the first study to explore in depth the biotechnological potential of bacterial cultures isolated from artisanal Croatian cheese as functional starter cultures for high-quality game meat sausage production.

Key words: fermented sausages, native starter cultures, dairy origin, rep-PCR, sensory properties

\section{INTRODUCTION}

Lactococci and enterococci are usually present in low numbers in artisanal dry fermented sausages and are considered as background microbiota with ambiguous impact on the physicochemical, sensorial and microbiological quality of the sausages (1-3). Although accepted as minor or even undesirable populations of lactic acid bacteria (LAB) in meat fermentation, particular strains of lactococci and enterococci possess specific and technologically interesting traits that could be used to improve the sausage production, especially sensorial acceptability $(2,3)$. Of all microbial metabolic pathways, those involved in carbohydrate, lipid and protein conversion contribute largely to the safety, overall 
acceptability and sensorial properties of the final products. Exogenous proteases and lipases have been successfully used to accelerate the ripening of dry fermented sausages, with the primary aim of reducing production costs (4). Likewise, proteolytic and lipolytic strains may significantly influence the ripening process and the quality of the ready-to-eat sausages. However, the final result is difficult to predict as, under certain conditions, excess proteolysis and lipolysis may result in bitter and metallic off-flavour due to the presence of bitter peptides or excess oxidation of lipids (5).

Spontaneously fermented sausages prepared from wild boar (Sus scrofa) hold an important place in the game meat production and consumption. They are generally characterized by distinctive sensory properties, differing from those of 'domestic' meat and are mostly darker, stronger tasting and often tougher, reflecting the fact that these animals are raised in the wild and feed on naturally growing plants $(6,7)$. Spontaneously fermented sausages are mostly manufactured by local producers following traditional procedures, without nitrate or starter culture addition. Consequently, they are recognized and appreciated as authentic traditional products, and their production is anticipated to increase as consumers demand more organic food of particular taste and flavour.

Although dry fermented sausages are considered microbiologically stable, in the case of elevated numbers of pathogens in raw material or inadequate production conditions, their safety can be compromised $(8,9)$. Several zoonotic agents and enteric bacteria can be transmitted from wildlife to humans by contaminated game meat, particularly if the intestine is ruptured by shot pellets or during evisceration. The risk especially increases under poorly controlled environmental conditions or if small game meat producers do not follow good manufacturing practice. However, to provide high-quality products and possibly expand the production of traditional meat sausages, high food safety standards have to be met. The possibilities are being investigated intensively, and frequently involve the use of starter cultures with proven antimicrobial and technological properties that might ensure both, microbiological safety and unimpaired sensorial quality of nitrate-free sausages (10-12). Besides, in terms of functional food, additional properties of starters such as probiotic potential are much appreciated (13). However, if potential starter culture does not multiply inside the meat product or/and is outcompeted by wild microbiota, its metabolic activity in sausages is minimized, despite in vitro potential and high performance under laboratory conditions (14).

As fermentation is an integral feature of both, dry sausage and cheese production, this has led to the evaluation of bacterial cultures Lactococcus lactis ssp. cremoris (LL8307) and Enterococcus durans (ED0207) originally isolated from artisanal Croatian hard type cheese to diversify the flavour range of dry fermented sausages and to increase their microbiological safety. Both strains were chosen for their high or medium acidifying, proteolytic and/or lipolytic activity and bioprotective potential after step-by-step selection of wild isolates.
Therefore, this study aims to evaluate the survival rate of selected starter cultures in wild boar meat sausages during the ripening period of 40 days at a local small-scale facility under artisanal conditions. Moreover, their influence on the microbiological, physicochemical and sensory properties as well as histamine content of sausages was determined.

\section{MATERIALS AND METHODS}

\section{Origin and strain selection}

All tested bacterial cultures were isolated from spontaneously fermented traditional Croatian cheese in a previous study (15) and deposited in the culture collection of the Department of Microbiology, Faculty of Agriculture, University of Zagreb. All Gram-positive, coagulase-negative cocci prevously identified as Enterococcus durans and Lactococcus lactis $(N=23)$ were subjected to a step-by-step selection including a detailed safety, technological and antimicrobial analyses.

\section{Safety, technological, antimicrobial and probiotic potential}

Firstly, the strain $(N=23)$ safety traits including the haemolysis, susceptibility to antibiotics as well as histamine and virulence determinants were determined. The haemolytic capacity of the strains was analyzed on Columbia blood agar (bioMérieux, Crappone, France) where Bacillus cereus DSM 6791 served as a positive control. The susceptibility to clinically relevant antibiotics including ampicillin ( 2 and $10 \mu \mathrm{g})$, clindamycin $(2 \mu \mathrm{g})$, gentamicin $(10 \mu \mathrm{g})$, tetracycline (5 and $10 \mu \mathrm{g})$, erythromycin ( 2 and $15 \mu \mathrm{g})$, vancomycin $(5 \mu \mathrm{g})$ and chloramphenicol $(30 \mu \mathrm{g})$ was measured by the standardized agar disc diffusion method using $\mathrm{BBL}^{\mathrm{TM}}$ Sensi-Disc ${ }^{\mathrm{TM}}$ antimicrobial susceptibility test discs (Becton, Dickinson and Company, Rungis, France) as described elsewhere (16). To detect the genes encoding for the production of histamine ( $h d c)$, a PCR assay was performed by following the protocol of de las Rivas et al. (17). Enterococci were additionally screened for the presence of virulence factors including aggregation substance (agg), gelatinase ( $g$ elE), cytolysin $(c y / M, c y / B)$, cytolysin activator (cylA), enterococcal surface protein (esp), and sex pheromones (cpd and $c o b$ ) as reported before (18).

The acidifying activity of selected strains was determined by measuring the $\mathrm{pH}$ in brain heart infusion (BHI) broth (Biolife, Milan, Italy) inoculated with an appropriate strain in duplicates. The measurements were taken at the beginning $(0 \mathrm{~h})$ and after $24 \mathrm{~h}$ of incubation at $37^{\circ} \mathrm{C}$ for enterococci and $30^{\circ} \mathrm{C}$ for lactococci. The $\mathrm{pH}$ was measured by combined $\mathrm{pH}$ electrodes (InPro ${ }^{\circledR} 3030 ;$ Mettler Toledo, Greifensee, Switzerland) that were disinfected after each use with $3 \% \mathrm{HCl}$. The acidification rate was calculated according to Jamaly et al. (19) and expressed as $\Delta \mathrm{pH}$.

Lipolytic activity was screened on tributyrin agar (Oxoid, Hampshire, UK) by disc diffusion method. Bacterial suspensions $(10 \mu \mathrm{L})$ at a cell concentration corresponding to the 0.5 
McFarland standard were inoculated on sterile cellulose discs (Bio-Rad Laboratories, Hercules, CA, USA) previously placed on the agar. Plates were then incubated for 3 days at $37^{\circ} \mathrm{C}$ for enterococci and $30^{\circ} \mathrm{C}$ for lactococci. Proteolytic activity was tested in the same way as lipolytic activity, except instead of tributyrin agar, $\mathrm{BHI}$ agar supplemented with skimmed milk (1.5\%) was used (16). The diameter of clear zone was measured and expressed as a mean value in $\mathrm{mm}$ and compared to the lypolitic and proteolytic activity of Pseudomonas fluorescens WCS $417 r$.

The peptidolytic activity was analyzed in the presence of chromogenic peptide $N$-succinyl-Ala-Ala-Pro-Phe- $p$-nitroanilide (Sigma-Aldrich, Merck, St Louis, MO, USA). The release of $p$-nitroanilide ( $p N A$ ) by the action of bacterial peptidases was measured at $410 \mathrm{~nm}$, as described by de Giori and Hébert (20). Results were expressed as $\mu \mathrm{M} p \mathrm{NA}$.

Antimicrobial activity was tested against seven indicator bacteria, Salmonella enterica (DSM 14221), Listeria innocua (ATCC 33090), Escherichia coli (ATCC 25922), Staphylococcus aureus ssp. aureus (DSM 20231), Brochotrix thermospachta (LMG 17208), Weissella viridescens (DSM 20410) and Bacillus cereus (DSM 6791) using a modified agar streak-spot technique (21). The difference between the colony diameter of indicator bacteria and the diameter of control colony was estimated. The results were expressed as follows: no inhibition $(<1 \mathrm{~mm})$, weak inhibition (1-2 mm), pronounced inhibition (2-4 mm), very strong inhibition ( $>4 \mathrm{~mm}$ ) and complete inhibition (no growth). The antimicrobial activity of strains was additionally tested using the cell-free supernatant neutralized with $1 \mathrm{M}$ $\mathrm{NaOH}$ and filtered through a $0.22-\mu \mathrm{m}$ membrane filter (Merck, Darmstadt, Germany) and the agar well diffusion method as described previously (22).

The probiotic potential of the best starter culture candidates Enterococcus durans (ED0207) and Lactococcus lactis ssp. cremoris (LL8307) with respect to safety, technological and antimicrobial traits was determined. In order to estimate the survival rate of strains in simulated gastric and intestinal conditions, the protocol of Doleyres et al. (23) with slight modifications as described by Mrkonjic Fuka et al. (16) was followed. Briefly, to simulate the gastric digestion, $0.5 \% \mathrm{NaCl}$ and $0.3 \%$ pepsin solution (Sigma Chemicals, Merck, St Louis, MO, USA) was used and the $\mathrm{pH}$ was adjusted to $\mathrm{pH}=2.5$ with $1 \mathrm{M} \mathrm{HCl}$. For cell survival under simulated intestinal conditions, $0.4 \%$ bile salt and $0.2 \%$ pancreatin solution (Sigma Chemicals, Merck) was applied. The survival of each strain was evaluated after the incubation under the above mentioned conditions and subsequent plating on $\mathrm{BHI}$ agar for colony forming units (CFU) count. Aggregation was evaluated as described by Del Re et al. (24) using $4 \mathrm{~mL}$ of cell suspension corresponding to viable counts of approx. $10^{8} \mathrm{CFU} / \mathrm{mL}$. The cell suspension was vortexed for $10 \mathrm{~s}$ and the absorbance was measured on UV/ Vis spectrophotometer (PerkinElmer, Waltham, MA, USA) at $610 \mathrm{~nm}$ after $5 \mathrm{~h}$ of incubation at room temperature.

In addition, the growth of potential starter cultures (ED0207 and LL8307) at temperatures relevant for artisan $\left(12{ }^{\circ} \mathrm{C}\right)$ and industrial $\left(25^{\circ} \mathrm{C}\right)$ sausage production and in the presence of typical stressors such as 3.0 and $6.0 \% \mathrm{NaCl}$ at $\mathrm{pH}=4.5$ was determined by visually assessing the turbidity after $48 \mathrm{~h}$ of incubation.

\section{Biomass preparation}

Before the application of potential starter cultures ED0207 and LL8307, they were grown aerobically in BHI broth $(100 \mathrm{~mL})$ at $30^{\circ} \mathrm{C}$ for $24 \mathrm{~h}$. Each strain was harvested by centrifugation at $8000 \times g$ for $5 \mathrm{~min}$. Cell pellets were then resuspended in $100 \mathrm{~mL}$ of sterile skimmed milk solution (1.5\%) and were added to the meat batter. Total viable count (TVC) of inoculum was estimated on plate count agar (PCA) (Merck) after $24 \mathrm{~h}$ of incubation at $30^{\circ} \mathrm{C}$.

\section{Formulation and sampling of sausages}

In this study, three batches of fermented sausages were prepared each with $25 \mathrm{~kg}$ of meat batter. All batches were made from a mixture of domestic pig (Sus scrofa domesticus L.) meat ( $50 \%$ ) and wild boar (Sus scrofa L.) meat (50\%) and the following ingredients were added: salt $(1.9 \%)$, red chilli peppers $(0.5 \%)$, garlic $(0.3 \%)$, red sweet peppers $(0.2 \%)$, sug$\operatorname{ar}(0.2 \%)$, and black pepper (0.1\%). Spices were added dried and ground. Selected native starter cultures were applied resuspended in sterile skimmed milk solution: E. durans ED0207 in treatment A, Lactococcus lactis ssp. cremoris LL8307 in treatment $B$, and treatment $C$ was used as a non-inoculated control (no starter cultures were added). Meat batter was filled in natural casings (pig's small intestine) with a $38 \mathrm{~mm}$ diameter, and the sausages were allowed to ripen in a drying chamber, with four smoking treatments under traditional conditions for 40 days. Sausages were randomly distributed in the fermentation/ripening chamber. Temperature and relative humidity were monitored every 30 min using data-logger LOG $32 \mathrm{TH}$ (Dostmann Electronic GMBH, Wertheim-Reicholzheim, Germany). Three sausages were sampled per one sampling time and analyzed independently at 0, 4, 7, 15 and 40 days for the evaluation of the survival rate, $\mathrm{pH}$, water activity and microbiological quality, and the final products were tested for histamine and sensory properties.

\section{Water activity and $\mathrm{pH}$}

Water activity $\left(a_{w}\right)$ was determined using a portable analyzer, HygroPalm HP23-AW-A equipped with an HC2-AW probe (Rotronic AG, Bassersdorf, Switzerland) and $\mathrm{pH}$ values were measured using a portable pH-meter IQ 150 (IQ Scientific Instruments, San Diego, CA, USA) equipped with a spear type glass electrode BlueLine 21pH (Schott AG, Mainz, Germany).

\section{Microbiological analysis of spontaneously fermented sausages}

Twenty-five grams of sample (without casing) of each sausage were aseptically transferred to sterile plastic pouches and homogenized in a sterile saline solution (0.85\%) using 
a Stomacher Lab-Blender 400 (Seward Medical, London, UK). Appropriate dilutions of the sample homogenates were prepared in duplicates in peptone water and inoculated in growth media for the enumeration and detection of particular microbial groups. Enterobacteriaceae were determined on Violet Red Bile Glucose agar (VRBG; Merck) after incubation at $37^{\circ} \mathrm{C}$ for $24 \mathrm{~h}$ according to ISO 21528-2:2004 (25). Staphylococcus aureus was determined on Baird Parker agar (Labo-Life Sàrl, Pully, Switzerland) supplemented with egg yolk tellurite emulsion (20\%; VWR International AG, Dieticon, Switzerland) and incubated at $37^{\circ} \mathrm{C}$ for $48 \mathrm{~h}$. Chromocult ${ }^{\oplus}$ Coliform agar ES (Merck) was used for the differentiation and enumeration of E. coli and coliforms at $37{ }^{\circ} \mathrm{C}$ for $24 \mathrm{~h}$, according to ISO 4832:2006 (26). Yeasts and moulds were enumerated on Dichloran Rose-Bengal Chloramphenicol agar (DRBC; Biolife, Milan, Italy) supplemented with chloramphenicol at $25^{\circ} \mathrm{C}$ for 5 days under aerobic conditions according to ISO 21527-2:2008 (27). Salmonella spp. and Listeria monocytogenes were detected according to ISO 6579:2002/AMD 1:2007 (28) and ISO 11290-1:1996/AMD 1:2004 (29), respectively. The presence of enterococci was determined on kanamycin esculin azide agar (KAA; Biolife) after incubation at $37^{\circ} \mathrm{C}$ for $48 \mathrm{~h}$. Lactobacillus spp. were isolated on MRS (de Man, Rogosa, and Sharpe, Merck) agar supplemented with vancomycin and bromocresol green (LAMVAB) (30) and Lactococcus spp. on M17 agar (Merck) under anaerobic conditions after $72 \mathrm{~h}$ at $30^{\circ} \mathrm{C}$. Approximately 15 colonies from the KAA and M17 media were randomly selected and purified from each spontaneously fermented sausage batch on sampling day $(0,7,15$ and 40 days; $N=150)$ and were subjected to DNA extraction.

\section{Extraction of DNA and identification of strains}

The template DNA was extracted from the KAA and M17 isolates following the protocol of Wizard Genomic DNA Purification Kit (Promega, Madison, WI, USA). The concentration and purity of the DNA were determined by a NanoDrop ND-1000 spectrophotometer (Thermo Fisher Scientific Inc., Waltham, MA, USA).

For the identification at the species level, part of the $16 \mathrm{~S}$ rRNA gene was amplified and sequenced (Macrogen, Seoul, South Korea) using the universal bacterial primer sets $27 \mathrm{f}$ and 1401R (31,32). The obtained sequences were analyzed with the Nucleotide Basic Local Alignment Search tool (BLASTn) (33) and a minimum sequence identity of $98 \%$ was chosen as a criterion for species identification.

In addition, all collected KAA and M17 isolates $(N=150)$ were identified at the strain level and genotyped to evaluate their survival efficiency during the ripening of sausages. The molecular fingerprinting and strain-specific identification were performed by the repetitive rep-PCR using (GTG) 5 oligonucleotide as a primer (34) as described elsewhere (21). The rep-PCR patterns were analyzed and compared to those obtained from the applied starter cultures Lactococcus lactis ssp. cremoris (LL8307) and Enterococcus durans (ED0207). Only $100 \%$ identical fingerprinting patterns were considered as the same strain.

\section{Histamine content}

An enzyme-linked immunosorbent assay (ELISA) kit for histamine determination (RIDASCREEN ${ }^{\circledR}$ ) was provided by R-Biopharm (Darmstadt, Germany). All reagents were included in the commercial kit and the assay was performed following the manufacturer's instructions.

\section{Sensory properties}

Sensory analysis was performed by a hedonic test using 112 consumers among students and faculty staff and the basic socio-demographic characteristics were as follows: $54 \%$ female and $46 \%$ male; $65 \%$ under age 35, $28 \%$ between 35 and 55 and $7 \%$ above age $55 ; 6 \%$ with low income, $70 \%$ with medium income and $24 \%$ with high income; $48 \%$ lived in rural and $52 \%$ in urban areas. Samples were scored on a 10-point structured scale where 0 meant 'extremely disliked' and 9 meant 'extremely liked'. Six sensory traits were evaluated: cross-section, odour, flavour, hardness, juiciness and overall likeability. Samples were cut by a knife to $2 \mathrm{~mm}$ thickness under $90^{\circ}$ angle, and the presentation order was defined as a completely balanced block design. Subjects were placed in separate booths and were instructed to use tap water and unsalted bread as palate cleansers before every sample.

\section{Statistical analysis}

The data were subjected to analysis of variance (one-way ANOVA) by SAS ${ }^{\circledR}$ Studio University Edition v. 3.8 (35) and Tukey's test with $p \leq 0.05$ was used for the evaluation of statistically significant differences. For the analysis of microbial count, histamine content, $\mathrm{pH}$ and water activity value, a general linear model with treatment as the fixed effect was used, while for the analysis of sensory data, the assessor was added as a random effect in a MIXED model. Results are presented as mean value \pm standard error.

\section{RESULTS AND DISCUSSION}

\section{Characterization of starter cultures}

In selecting strains with optimal properties, safety was the first selection criterion followed in our study. Although all testing isolates showed no haemolytic activity, most of them did not pass safety characterization ( $82 \%$ ) as they were resistant to one or more antibiotics (mostly tetracycline, clindamycin and rifampicin) or possessed more than one virulence determinants (data not shown). The remaining four candidates that passed the safety characterization were tested for acidifying, proteolytic and lipolytic activities as the most important technological properties of starter cultures in fermented meat products because of their influence on texture and flavour development (36). Of tested strains $(N=4)$, one was low acidifier $(\Delta \mathrm{pH}<1.5)$ and was discarded from further analysis, and three showed medium $(\Delta \mathrm{pH}=1.5-2.0)$ or high $(\Delta \mathrm{pH}>2.0)$ acidification potential (37). The lipolysis was noticed only for one of the three tested isolates. All three 
candidates showed some degree of proteolytic or peptidolytic activity; however, only one candidate stood out with high values considering proteolytic and peptidolytic potential. Based on the above results, two candidate strains Lactococcus lactis ssp. cremoris (LL8307) and Enterococcus durans (ED0207) were selected and further analyzed (Table 1). These strains exhibited a medium (ED0207; $\Delta \mathrm{pH}=1.50$ ) to high acidification potential (LL8307; $\Delta \mathrm{pH}=2.32)$, pronounced peptidolytic activity measured as concentration of $p$-nitroanilide ( $p N A)$ released from the chromogenic peptide (S-Ala) ((21578 239$)$ $\mu \mathrm{M}$ for LL8307), or noticeable lipolytic activity analyzed on agar with tributyrin (ED0207).

Both strains grew at temperatures usually used for traditional or industrial meat fermentation $\left(12\right.$ and $25^{\circ} \mathrm{C}$ ) and exhibited physiological traits characteristic for each bacterial group. Strain LL8307 did not grow at $6 \% \mathrm{NaCl}$ irrespective of the incubation temperature, and much reduced growth of both strains was noticed at $12{ }^{\circ} \mathrm{C}$, even resulting in the absence of growth at $\mathrm{pH}=4.5(\mathrm{ED0207})$ or in 3 or $6 \% \mathrm{NaCl}$ (LL8307).

The weak antimicrobial capacity ( $<1 \mathrm{~cm}$ diameter) of both strains was observed against Gram-positive and -negative bacteria. A somewhat broader spectrum of antimicrobial activity of strain ED0207 was noticed (against Salmonella spp., S. aureus, E. coli, Listeria innocua and Weisella viridescens) than of LL8307 (against Salmonella spp., S. aureus and Weisella viridescens). However, this activity was not detected using the cell-free supernatants, suggesting that the inhibitory effect was most probably a result of the production of antimicrobially active metabolites such as organic acids or hydrogen peroxide, rather than the ability to produce bacteriocins (14).

However, mechanisms underlying the activity of $L A B$ strains against bacterial pathogens appear to be multifactorial (38) and they are well known in the Gl tract. As such, LAB can prevent the adhesion of pathogens by competing for the binding sites on the intestinal epithelial cells and consequently, reduce the colonization, thereby preventing the onset of infection $(39,40)$. However, in order to extend beneficial effects in the intestine, besides a high initial level of viable microorganisms, a probiotic needs to achieve adequate biomass through growth and aggregation. Consequently, the ability to aggregate is a desirable property of probiotics (41).

Both tested strains in our study showed a similar surviving rate under gastric conditions, 65.31 and $56.89 \%$ for ED0207 and LL8307 respectively. However, remarkable differences $(p<0.01)$ were noticed under simulated duodenal conditions, where $81.70 \%$ of the added ED0207 and only $16.38 \%$ of LL8307 survived. However, despite the huge growth reduction of LL8307 in the presence of bile salt and pancreatin, more than $10^{8}$ cells (from the initial $2.9 \cdot 10^{9}$ ) still survived the passage through the duodenum, which should be adequate biomass to exhibit probiotic efficiency (42). However, the autoaggregation ability of both strains was either low ( $25 \%$ for LL8307) or absent (5\% for ED0207), meaning low or no capacity of adhesiveness and persistence in the GI tract (24).

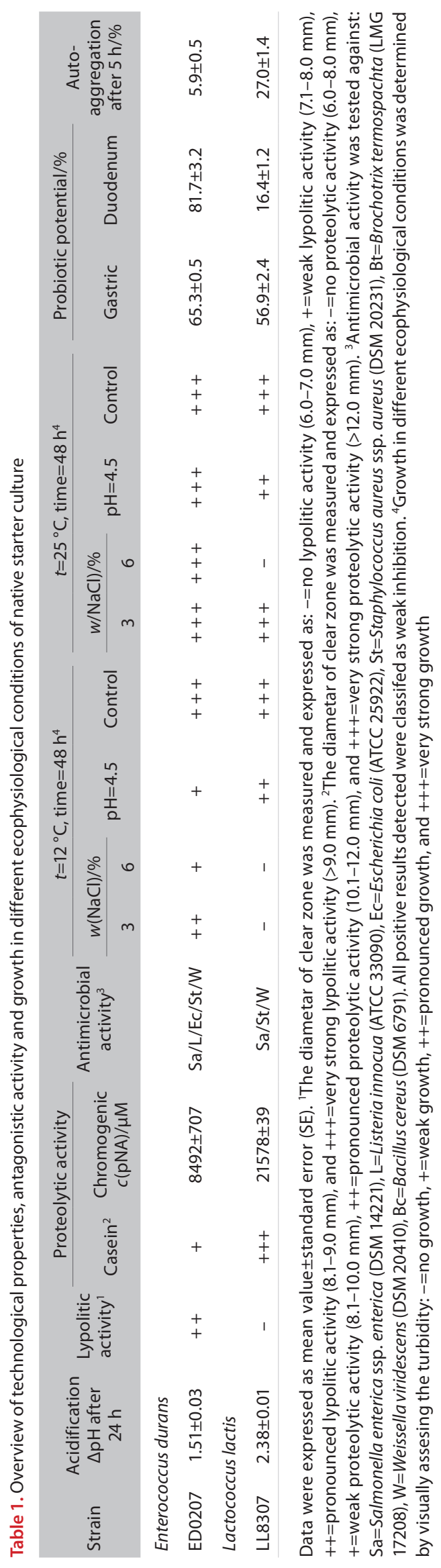


Biomass production and the survival rate of the inoculated starter cultures

To ensure that a starter culture is effective, appropriate bacterial biomass should be produced and applied to the meat batter. The level of culture to be added depends on the product specifications, but a high viable number of cells is generally used, ranging from 5 to $9 \log$ CFU/g (14). In this study, the viable cell counts of the inoculum added to the meat batches A and B were comparable: $(9.32 \pm 0.06)$ log CFU/ $\mathrm{mL}$ of ED0207 (batch A) and (9.38 \pm 0.08 ) $\log$ CFU/mL of LL8307 (batch B), corresponding to the 6.98 and $6.92 \mathrm{log}$ CFU/g of meat respectively.

However, the ability of the starter culture to compete with the natural microbiota and to undertake the metabolic activities to improve the nutritional and microbiological quality of fermented sausages is based on its ability to multiply inside the meat product, not only on high biomass applied (14). Therefore, to follow the succession of a particular strain in a complex microbial community, rep-PCR fingerprinting of KAA and M17 isolates on days $0,7,15$ and 40 was performed. Based on the rep-PCR analysis, a different pattern for the survival rate of the applied starters was noticed (Fig. 1). On day seven, a $10 \%$ reduction of survival rate of both strains was observed. However, a remarkable difference was noticed on day 15 when less than $33 \%$ of isolates were assigned to ED0207 and $80 \%$ to LL8307. On day 40, only $20 \%$ of enterococcal isolates were identified as ED0207 and none of the LL8307 was detected. At the same time, the number of both strains and on both days was higher than 5.2 log $\mathrm{CFU} / \mathrm{g}$, which led to the conclusion that wild enterococci and lactococci outcompeted the added starter cultures during the prolonged ripening period. Moreover, in many similar

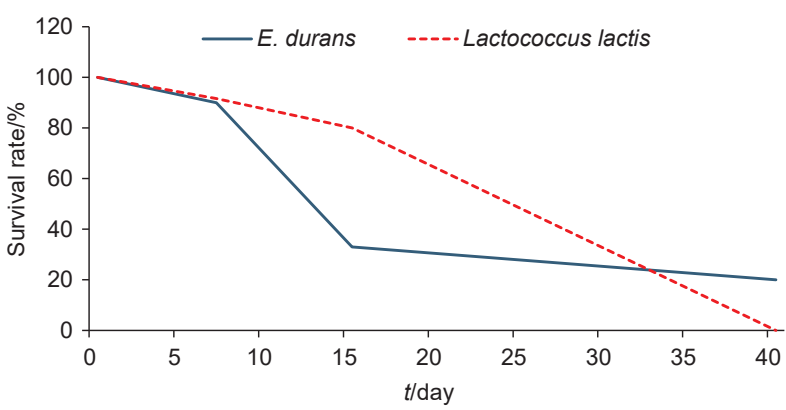

Fig. 1. Survival rate of applied starter cultures Enterococcus durans ED0207 and Lactococcus lactis ssp. cremoris LL8307

studies, the survival rate of the applied starter culture was followed by counting microbes on selective agar media typical for a particular microbial group $(43,44)$. Because of the findings in our study, such an approach is not satisfactory and should be always conducted with a relevant fingerprinting method that allowed tracking of the particular strain of interest.

\section{Microbial evolution, physicochemical properties and histamine content}

The whole process of sausage production was closely monitored but only partly controlled as it is usually performed in artisanal production. Three distinctive temperatures and relative humidity values were characterized (Fig. 2). During the first five days, the temperature varied from 5.5 to $22^{\circ} \mathrm{C}$ (with four peaks during smoking) and relative humidity was between 54 and $87 \%$ (lower during the smoking phase). In the next 21 days, the temperature was between 5 and $12^{\circ} \mathrm{C}$ and relative humidity between 74 and $90 \%$. In the third period, the temperature was affected by the extreme outer conditions, with lower values close to $1{ }^{\circ} \mathrm{C}$ and the maximum was $8{ }^{\circ} \mathrm{C}$. The third period was also characterized by very stable but high relative humidity (79-90\%), not usually present in artisanal production. These data show that artisanal production, which is affected by outer weather conditions, is variable, as previously found by Zgomba Maksimovic et al. (8), and this highlights the need for standardization in other aspects like raw material and ingredient quality and/or starter cultures application.

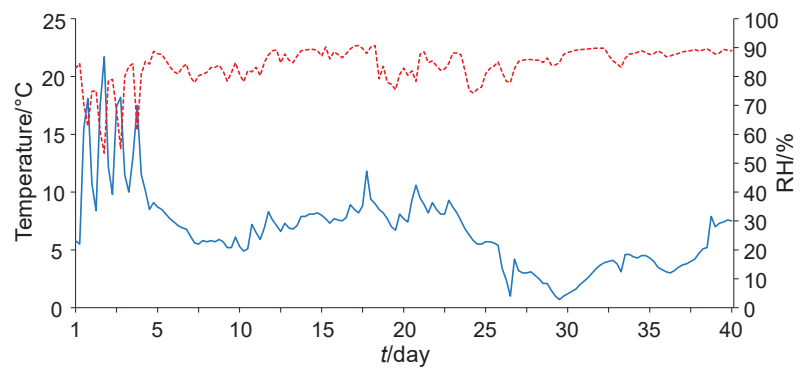

Fig. 2. Temperature (blue line) and relative humidity ( $\mathrm{RH}$, red line) during sausage production

The mass of sausages was on average $544.50 \mathrm{~g}$, and the production ended when mass loss reached about $30 \%$. Water activity $\left(a_{\mathrm{w}}\right)$ and $\mathrm{pH}$ were measured at different times of the production (Table 2) and the change of $a_{\mathrm{w}}$ and $\mathrm{pH}$ values was typical for fermented artisanal wild boar sausages $(8,45)$. At the beginning of production, the $a_{w}$ was between 0.97 and 0.98 in all three treatments, which slightly declined after seven days (0.93-0.95) and reached values between 0.86 and 0.87 in the final products (Table 2). The significant differences in $a_{w}$ between treatments with starter cultures and control samples were found only on days 7 and 15 of production ( $p>0.05$ ). A similar trend was detected for $\mathrm{pH}$ values, which were uniform at the beginning and the end of production. Significant differences between control and experimental treatments were established on day 7 , when the effect of starter culture addition was seen as lower $\mathrm{pH}$ values. These results correspond to the $a_{w}$ values that were significantly lower in control treatment on day 7 than in experimental treatments (0.93 vs 0.95), meaning that microbial growth is 


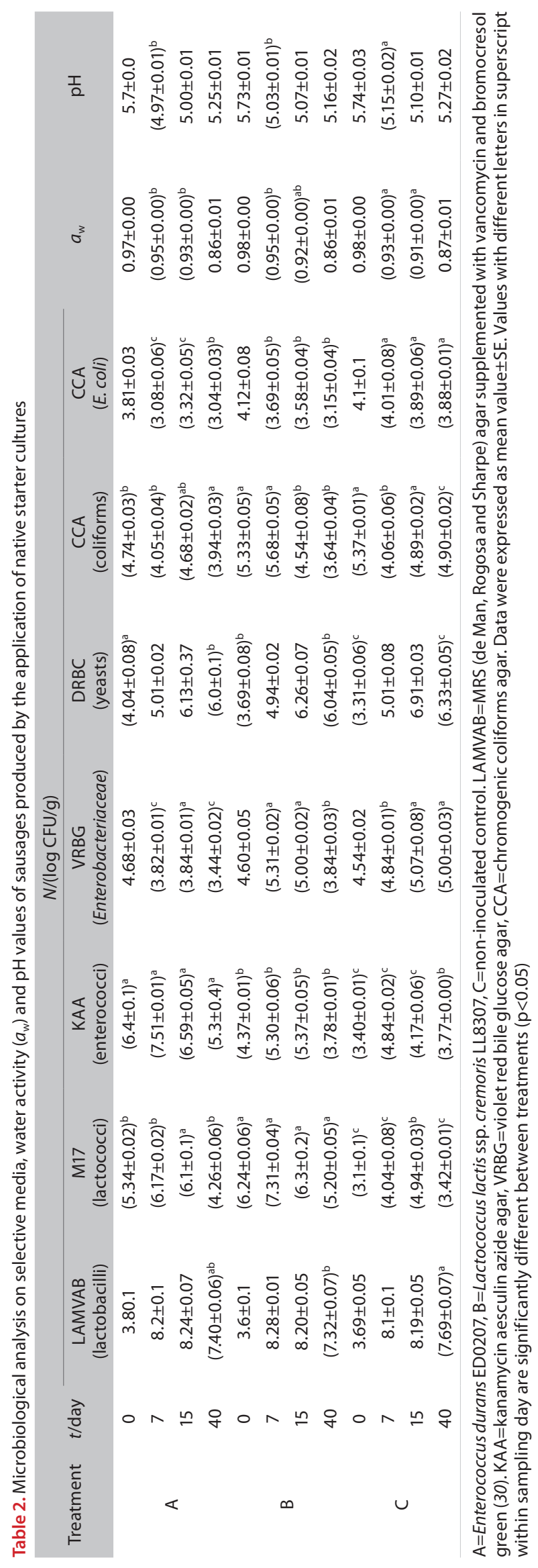

slightly reduced in the control treatment, resulting in the reduced formation of organic acids.

The number of lactobacilli isolated on the LAMVAB medium on day zero was comparable in all three treatments (3.69-3.75 log CFU/g). A similar trend was noticed during the fermentation and ripening indicating that applied starter cultures did not affect the growth of wild lactobacilli in our trial. The number of lactobacilli reached the level above 8 log $\mathrm{CFU} / \mathrm{g}$ in all treatments and remained there until the end of ripening, when it decreased to 7.32-7.69 $\log$ CFU/g $(p<0.05)$ (Table 2).

Although Salmonella spp., Staphylococcus aureus and Listeria monocytogenes were not detected, the elevated number of coliforms, E. coli and Enterobacteriaceae is of special concern. The initial high number of respective microbes (above $4 \mathrm{log}$ CFU/g of Enterobacteriaceae or E. coli and above $5 \mathrm{log}$ CFU/g of coliforms) was only partially overcome by the application of starter cultures. Although a significantly lower number $(p<0.05)$ of coliforms, E. coli and Enterobacteriaceae was noticed in ready-to-eat sausages produced with starter cultures than in the control, the amount of respective microbial groups still exceeded the limits set by the Health Protection Agency (HPA) (46) in all samples, except in treatment B (Table 2). The findings above suggest poor raw meat hygiene, most probably due to the faecal contamination at shooting or evisceration. As such, the power of starter cultures to decrease the number of undesirable microbiota to acceptable values is much reduced when an elevated number of potential pathogens is present in the raw material.

The content of biogenic amines in sausages can be influenced by a combination of many factors, such as ripening conditions, formulation, $\mathrm{pH}$, temperature, additives, diameter and salt/water ratio, as well as the proteolytic and decarboxylase activities of developed microbiota (47). Although there is no legislation dealing with the content of histamine in dry fermented sausages, the mass fraction between 100 and 200 $\mathrm{mg} / \mathrm{kg}$ seems to be acceptable for many countries (48). However, the histamine mass fraction measured in our study was far below these values and comparable in all three treatments: $3.31,4.27$ and $4.54 \mathrm{mg} / \mathrm{kg}$ in treatment $\mathrm{A}, \mathrm{C}$ and B, respectively.

\section{Sensorial quality}

Analysis of hedonic test data revealed that sensory traits of sausages did not differ significantly between treatments, as shown in Table 3. This is similar to the research conducted by Talon et al. (49) who found that the addition of autochthonous starters did not affect the overall aroma and flavour of the sausages, but there were some effects on texture traits. Contrary, the preference ranking test (45) revealed that the addition of bacterial starter cultures had a significant and positive effect on the sensory score. Within this research, all sensory traits had an average value around 7, except hardness, which was rated with lower values. Because average values were similar, frequency analysis was performed and 
Table 3. Sensory traits of sausages produced by the addition of native culture starters

\begin{tabular}{lccc} 
& \multicolumn{3}{c}{ Treatment } \\
\cline { 2 - 4 } Trait & $\mathrm{A}$ & $\mathrm{B}$ & $\mathrm{C}$ \\
Cross-section & $7.0 \pm 0.2$ & $7.0 \pm 0.1$ & $7.1 \pm 0.1$ \\
Odour & $7.2 \pm 0.2$ & $7.3 \pm 0.1$ & $7.1 \pm 0.1$ \\
Flavour & $7.2 \pm 0.1$ & $6.9 \pm 0.2$ & $7.1 \pm 0.2$ \\
Hardness & $6.71 \pm 0.21$ & $6.3 \pm 0.2$ & $6.6 \pm 0.2$ \\
Juiciness & $7.07 \pm 0.14$ & $7.4 \pm 0.1$ & $7.2 \pm 0.1$ \\
Overall likeability & $7.2 \pm 0.1$ & $7.2 \pm 0.1$ & $7.2 \pm 0.1$
\end{tabular}

$\mathrm{A}=$ Enterococcus durans ED0207, B=Lactococcus lactis ssp. cremoris LL8307, C=non-inoculated control

Data were expressed as mean value \pm SE

scores equal to or higher than 7 were counted (Fig. 3). This was described as the percentage of consumers scoring sensory traits with high likeability. It was established that all traits were highly liked by more than $50 \%$ consumers with hardness being the least liked, and overall likeability being the most liked trait. There were some differences between treatments and traits. Some of the traits (odour, flavour, juiciness) of experimental treatments $A$ and $B$ were highly liked by the higher percentage of consumers, suggesting the positive contribution of starter cultures to sensory traits. This can lead to the conclusion that the addition of starter cultures did not have any adverse effect on sensory traits and can even improve likeability in some groups of consumers. It is well known that the use of well-selected strains that generate high amounts of aroma components could improve the sensory quality and/or accelerate the meat fermentation process, as stated by Leroy et al. (50). The application of these high-yielding starters was not performed in this investigation, and a significant effect on sensory traits was not established. This can be further elaborated with the addition of a large amount of intensive spices (garlic and red hot pepper) into meat batter, which can dominate the aroma profile. Finally, the sensory hedonic testing was performed on a large number of untrained consumers ( $N=112)$ who usually increase variability and are unwilling to give maximum scores like trained panellists.

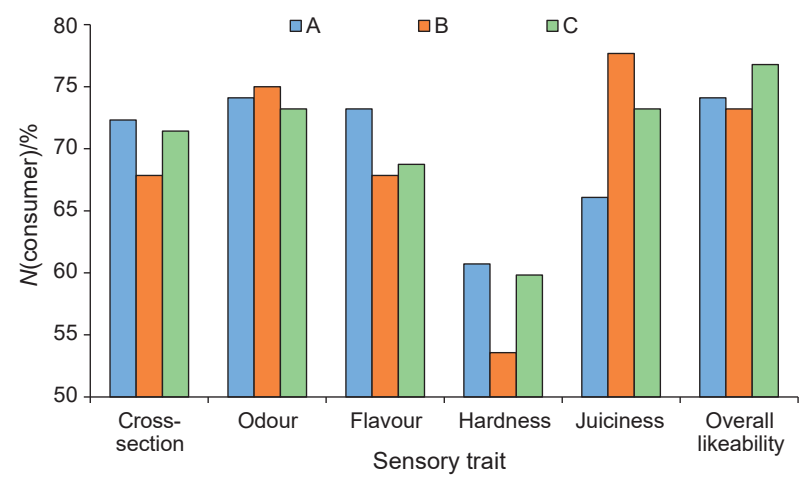

Fig. 3. Percentage of consumers scoring sensory traits with high likeability. $A=$ Enterococcus durans ED0207, B=Lactococcus lactis ssp. cremoris LL8307, C=non-inoculated control

\section{CONCLUSIONS}

In this study safety, biotechnological and probiotic properties of twenty-three enterococcal and lactococcal isolates of dairy origin were analyzed. Based on the obtained results, two best candidates showing high or medium acidifying, proteolytic and/or lipolytic activity as well as bioprotective and probiotic potential were selected and applied into the meat batter and evaluated for their survival rate and effect on physicochemical, microbiological and sensorial properties as well as histamine content of artisanal wild boar meat sausages.

The present study demonstrates that strains of lactococci and enterococci of dairy origin might be able to inhibit potential pathogens and improve sensory properties, and at the same time maintain the final $\mathrm{pH}$ within the range for non-acid/low-acid fermented sausages. However, the poor hygienic quality of meat can only partially be overcome by the application of starter cultures. The high hygienic quality of meat must be therefore the standard goal for high-quality sausage production.

Both applied native starter cultures survived in the sausages until day 15 of production; however, they were either absent from or present in a very low number in final products, which leads to the conclusion that wild enterococci and lactococci outcompete our starter strains and, consequently, their probiotic features can be excluded. Finally, this points to the need for tracking of specific strains in starter formulation by fingerprinting method through the whole process of production, as counting of a particular microbial group on selective agar media is not sufficient.

\section{FUNDING}

This work was supported by the Croatian Ministry of Agriculture as part of the project 'Application of microbial cultures in order to improve the technology of production of traditional wild boar sausages' (VIP-05092013).

\section{AUTHORS' CONTRIBUTION}

MMF designed the study, interpreted data and wrote the manuscript. IK designed and performed sensory and physicochemical analyses, participated in statistical data analysis and prepared figures. AZM prepared microbial inoculum, performed classical microbiological analysis and took part in data analysis. MB isolated DNA and performed fingerprinting of isolates. IT critically reviewed the manuscript, participating in data interpretation and prepared tables.

\section{CONFLICT OF INTEREST}

The authors declare that there is no conflict of interest.

\section{ORCID ID}

M. Mrkonjic Fuka (1) https://orcid.org/0000-0002-8494-8805

I. Kos $\odot$ https://orcid.org/0000-0002-2126-2566 
A. Zgomba Maksimovic ㄴo https://orcid.org/0000-0003-20768562

M. Bacic (1) https://orcid.org/0000-0002-5434-8251

I. Tanuwidjaja ㄴ) https://orcid.org/0000-0003-1364-2953

\section{REFERENCES}

1. Santos SC, Fraqueza MJ, Elias M, Barreto AS, Semedo-Lemsaddek T. Traditional dry smoked fermented meat sausages: Characterization of autochthonous enterococci. LWT - Food Sci Technol. 2017;79:410-5.

https://doi.org/10.1016/j.lwt.2017.01.042

2. Dowdell P, Chankhamhaengdecha S, Panbangred W, Janvilisri T, Aroonnual A. Probiotic activity of Enterococcus faecium and Lactococcus lactis isolated from Thai fermented sausages and their protective effect against Clostridium difficile. Probiotics Antimicrob Proteins. 2020;12:641-8. https://doi.org/10.1007/s12602-019-09536-7

3. Frece J, Kovačević D, Kazazić S, Mrvčić J, Vahčić N, Ježek D, et al. Comparison of ensory properties, shelf-life and microbiological safety of industrial sausages produced with autochthonous and commercial starter cultures. Food Technol Biotechnol. 2014;52(3):307-16.

4. Fernández M, Ordónez JA, Bruna JM, Herranz B, de la Hoz $L$. Accelerated ripening of dry fermented sausages. Trends Food Sci Technol. 2000;11(6):201-9. https://doi.org/10.1016/S0924-2244(00)00077-7

5. Toldrá F. Biotechnology of flavor generation in fermented meats. In: Toldrá F, editor. Meat biotechnology. New York, NY, USA: Springer; 2008. pp. 199-215. https://doi.org/10.1007/978-0-387-79382-5_9

6. Soriano A, Cruz B, Gómez L, Mariscal C, Ruiz AG. Proteolysis, physicochemical characteristics and free fatty acid composition of dry sausages made with deer (Cervus elaphus) or wild boar (Sus scrofa) meat: A preliminary study. Food Chem. 2006;96(2):173-84.

https://doi.org/10.1016/j.foodchem.2005.02.019

7. Utrilla MC, Ruiz AG, Soriano A. Effect of partial reduction of pork meat on the physicochemical and sensory quality of dry ripened sausages: Development of a healthy venison salchichon. Meat Sci. 2014;98(4):785-91.

https://doi.org/10.1016/j.meatsci.2014.07.027

8. Zgomba Maksimovic A, Zunabovic-Pichler M, Kos I, Mayrhofer S, Hulak N, Domig KJ, Mrkonjic Fuka M. Microbiological hazards and potential of spontaneously fermented game meat sausages: A focus on lactic acid bacteria diversity. LWT - Food Sci Technol. 2018;89:418-26.

https://doi.org/10.1016/j.lwt.2017.11.017

9. Marty E, Buchs J, Eugster-Meier E, Lacroix C, Meile L. Identification of staphylococci and dominant lactic acid bacteria in spontaneously fermented Swiss meat products using PCR-RFLP. Food Microbiol. 2012;29(2):157-66.

https://doi.org/10.1016/j.fm.2011.09.011
10. Frece J, Markov K, Kovačević D. Determination of indigenous microbial populations, mycotoxins and characterization of potential starter cultures in Slavonian kulen. Meso. 2010;12(2):92-9.

11. Gao Y, Li D, Liu X. Bacteriocin-producing Lactobacillus sakei C2 as starter culture in fermented sausages. Food Control. 2014;35(1):1-6.

https://doi.org/10.1016/j.foodcont.2013.06.055

12. Xie C, Wang HH, Nie XK, Chen L, Deng SL, Xu XL. Reduction of biogenic amine concentration in fermented sausage by selected starter cultures. CYTA - J Food. 2015;13(4):491-7. https://doi.org/10.1080/19476337.2015.1005027

13. Syngai GG, Gopi R, Bharali R, Dey S, Lakshmanan GMA, Ahmed G. Probiotics - The versatile functional food ingredients. J Food Sci Technol. 2016;53(2):921-33. http://doi.org/10.1007/s13197-015-2011-0

14. Oliveira M, Ferreira V, Magalhães R, Teixeira P. Biocontrol strategies for Mediterranean-style fermented sausages. Food Res Int. 2018;103:438-49.

https://doi.org/10.1016/j.foodres.2017.10.048

15. Mrkonjić Fuka M, Wallisch S, Engel M, Welzl G, Havranek J, Schloter M. Dynamics of bacterial communities during the ripening process of different Croatian cheese types derived from raw ewe's eilk cheeses. PLoS ONE. 2013;8(11): e80734.

https://doi.org/10.1371/journal.pone.0080734

16. Mrkonjic Fuka M, Zgomba Maksimovic A, Tanuwidjaja I, Hulak N, Schloter M. Characterization of enterococcal community isolated from an artisan Istrian raw milk cheese: $\mathrm{Bi}$ otechnological and safety aspects. Food Technol Biotechnol. 2017;55(3):368-80.

https://doi.org/10.17113/ftb.55.03.17.5118

17. de las Rivas B, Marcobal Á, Muñoz R. Improved multiplex-PCR method for the simultaneous detection of food bacteria producing biogenic amines. FEMS Microbiol Lett. 2005;244(2):367-72.

https://doi.org/10.1016/j.femsle.2005.02.012

18. Eaton TJ, Gasson JM. Molecular screening of Enterococcus virulence determinants and potential for genetic exchange between food and medical isolates. Appl Environ Microbiol. 2001;67(4):1628-35.

https://doi.org/10.1128/AEM.67.4.1628-1635.2001

19. Jamaly N, Benjouad A, Comunian R, Daga E, Bouksaim M. Characterization of enterococci isolated from Moroccan dairy products. Afr J Microbiol Res. 2010;4(16):1768-74.

20. de Giori GS, Hébert EM. Methods to determine proteolytic activity of lactic acid bacteria. In: Spencer JFT, de Ragout Spencer AL, editors. Food microbiology protocols. Methods in biotechnology, vol 14. Totowa, New Jersey, USA: Humana Press; 2001. pp. 197-202.

https://doi.org/10.1385/1-59259-029-2 
21. Domig KJ, Kiss H, Petricevic L, Viernstein H, Unger F, Kneifel W. Strategies for the evaluation and selection of potential vaginal probiotics from human sources: An exemplary study. Benef Microbes. 2014;5(3):263-72.

https://doi.org/10.3920/BM2013.0069

22. Kim JW, Rajagopal SN. Antibacterial activities of Lactobacillus crispatus ATCC 33820 and Lactobacillus gasseri ATCC 33323. J Microbiol. 2001;39(2):146-8.

23. Doleyres Y, Fliss I, Lacroix C. Increased stress tolerance of Bifidobacterium longum and Lactococcus lactis produced during continuous mixed-strain immobilized-cell fermentation. J Appl Microbiol. 2004;97(3):527-39.

https://doi.org/10.1111/j.1365-2672.2004.02326.x

24. Del Re B, Sgorbati B, Miglioli M, Palenzona D. Adhesion, autoaggregation and hydrophobicity of 13 strains of Bifidobacterium longum. Lett Appl Microbiol. 2000;31(6):438-42. https://doi.org/10.1046/j.1365-2672.2000.00845.x

25. ISO 21528-2:2004. Microbiology of food and animal feeding stuffs - Horizontal methods for the detection and enumeration of Enterobacteriaceae - Part 2: Colony count method. Geneva, Switzerland: International Organization for Standardization (ISO); 2004.

26. ISO 4832:2006. Microbiology of food and animal feeding stuffs - Horizontal method for the detection and enumeration of coliforms - Colony-count technique. Geneva, Switzerland: International Organization for Standardization (ISO); 2006.

27. ISO 21527-2:2008. Microbiology of food and animal feeding stuffs - Horizontal method for the enumeration of yeast and moulds - Part 2: Colony count technique in products with water activity less than or equal to 0.95 . Geneva, Switzerland: International Organization for Standardization (ISO); 2008.

28. ISO 6579:2002/AMD 1:2007. Microbiology of food and animal feeding stuffs - Horizontal method for the detection of Salmonella spp. - Amendment 1: Annex D: Detection of Salmonella spp. in animal faeces and in environmental samples from the primary production stage. Geneva, Switzerland: International Organization for Standardization (ISO); 2007.

29. ISO 11290-1:1996/AMD 1:2004. Microbiology of food and animal feeding stuff-Horizontal method for the detection and enumeration of Listeria monocytogenes - Part 1: Detection method - Amendment 1: Modification of the isolation media and the haemolysis test, and inclusion of precision data. Geneva, Switzerland: International Organization for Standardization (ISO); 2004.

30. Hartemink R, Domenech VR, Rombouts FM. LAMVAB - A new selective medium for the isolation of lactobacilli from faeces. J Microbiol Methods. 1997;29(2):77-84. https://doi.org/10.1016/S0167-7012(97)00025-0

31. Lane DJ. $16 \mathrm{~S} / 23 \mathrm{~S}$ rRNA sequencing. In: Stackebrandt $E$, Goodfellow M, editors. Nucleic acid techniques in bacterial systematics. Chichester, New York, USA: Wiley; 1991. pp. 115-75.

32. Nübel U, Engelen B, Felske A, Snaidr J, Wieshuber A, Amann RI, et al. Sequence heterogeneities of genes encoding $16 \mathrm{~S}$ rRNAs in Paenibacillus polymyxa detected by temperature gradient gel electrophoresis. J Bacteriol. 1996;178(19):5636-43.

https://doi.org/10.1128/JB.178.19.5636-5643.1996

33. Altschul SF, Gish W, Miller W, Myers EW, Lipman DJ. Basic local alignment search tool. J Mol Biol. 1990;215(3):403-10. Available from: https://blast.ncbi.nlm.nih.gov. https://doi.org/10.1016/S0022-2836(05)80360-2

34. Versalovic J, Schneider M, De Bruijn FJ, Lupski JR. Genomic fingerprinting of bacteria using repetative sequence-based polymerase chain reaction. Methods Mol Cell Biol. 1994;5: 25-40.

35. SAS ${ }^{\circledR}$ Studio University Edition, v. 3.8, User's Guide, SAS Institute Inc, Cary, North Carolina, USA; 2018. Available from: https://documentation.sas.com/?docsetld=webeditorug\&docsetTarget=titlepage.htm\&docsetVersion=3.8\&locale $=$ en.

36. Drosinos EH, Paramithiotis S, Kolovos G, Tsikouras I, Metaxopoulos I. Phenotypic and technological diversity of lactic acid bacteria and staphylococci isolated from traditionally fermented sausages in Southern Greece. Food Microbiol. 2007;24(3):260-70.

https://doi.org/10.1016/j.fm.2006.05.001

37. Sarantinopoulos $P$, Andrighetto C, Georgalaki MD, Rea MC, Lombardi A, Cogan TM, et al. Biochemical properties of enterococci relevant to their technological performance. Int Dairy J. 2001;11(8):621-47.

https://doi.org/10.1016/S0958-6946(01)00087-5

38. Campana R, Van Hemert S, Baffone W. Strain-specific probiotic properties of lactic acid bacteria and their interference with human intestinal pathogens invasion. Gut Pathog. 2017;9:12.

https://doi.org/10.1186/s13099-017-0162-4

39. Garriga M, Rubio R, Aymerich T, Ruas-Madiedo P. Potentially probiotic and bioprotective lactic acid bacteria starter cultures antagonise the Listeria monocytogenes adhesion to HT29 colonocyte-like cells. Benef Microbes. 2015;6(3): 337-43.

https://doi.org/10.3920/BM2014.0056

40. Abdel-Daim A, Hassouna N, Hafez M, Ashor MSA, Aboulwafa MM. Antagonistic activity of Lactobacillus Isolates against Salmonella typhi in vitro. Biomed Res Int. 2013;2013: 680605.

https://doi.org/10.1155/2013/680605

41. Satyanarayana T, Johri BN, Das SK, editors. Microbial diversity in ecosystem sustainability and biotechnological applications, vol. 1. Microbial diversity in normal \& extreme environments. Singapore: Springer; 2019.

https://doi.org/10.1007/978-981-13-8315-1 
42. Weese JS, Martin H. Assessment of commercial probiotic bacterial contents and label accuracy. Can Vet J. 2011;52(1): 43-6.

43. Palavecino Prpich NZ, Castro MP, Cayré ME, Garro OA, Vignolo GM. Autochthonous starter culture selection to keep traditions in the manufacture of dry sausages alive. Ann Microbiol. 2015;65(3):1709-19.

https://doi.org/10.1007/s13213-014-1010-0

44. Cenci-Goga BT, Rossitto PV, Sechi P, Parmegiani S, Cambiotti V, Cullor JS. Effect of selected dairy starter cultures on microbiological, chemical and sensory characteristics of swine and venison (Dama dama) nitrite-free dry-cured sausages. Meat Sci. 2012;90(3):599-606.

http://doi.org/10.1016/j.meatsci.2011.09.022

45. Paulsen P, Vali S, Bauer F. Quality traits of wild boar mould-ripened salami manufactured with different selections of meat and fat tissue, and with and without bacterial starter cultures. Meat Sci. 2011;89(4):486-90. http://doi.org/10.1016/j.meatsci.2011.05.014

46. Health Protection Agency. Guidelines for assessing the microbiological safety of ready-to-eat foods placed on the market. London, UK: Health Protection Agency; 2009. Available from: https://assets.publishing.service.gov.uk/government/uploads/system/uploads/attachment_data/file/ 363146/Guidelines_for_assessing_the_microbiological_ safety_of_ready-to-eat_foods_on_the_market.pdf.

47. Latorre-Moratalla ML, Bover-Cid S, Aymerich T, Marcos B, Vidal-Carou MC, Garriga M. Aminogenesis control in fermented sausages manufactured with pressurized meat batter and starter culture. Meat Sci. 2007;75(3):460-9.

https://doi.org/10.1016/j.meatsci.2006.07.020

48. Karovičová J, Kohajdová Z. Biogenic amines in food. Chem Pap. 2005;59(1):70-9.

49. Talon R, Leroy S, Lebert I. Microbial ecosystems of traditional fermented meat products: The importance of indigenous starters. Meat Sci. 2007;77(1):55-62.

https://doi.org/10.1016/j.meatsci.2007.04.023

50. Leroy F, Verluyten J, De Vuyst L. Functional meat starter cultures for improved sausage fermentation. Int J Food Microbiol. 2006;106(3):270-85.

https://doi.org/10.1016/j.ijfoodmicro.2005.06.027 